\title{
Calculation of the piezomagnetic field arising from uniform regional stress in inhomogeneously magnetized crust (II): Limitation in general cases
}

\author{
Ken'ichi Yamazaki ${ }^{1.2}$ \\ ${ }^{1}$ Institute of Seismology and Volcanology, Hokkaido University, N12-W8, Kita-ku, Sapporo, Hokkaido 060-0810, Japan \\ ${ }^{2}$ Miyazaki Observatory, Research Center for Earthquake Prediction, Disaster Prevention Research Institute, Kyoto University, \\ 3884, Kaeda, Miyazaki 889-2161, Japan
}

(Received August 17, 2010; Revised June 14, 2011; Accepted August 5, 2011; Online published February 21, 2012)

\begin{abstract}
One of the difficulties encountered in modeling the piezomagnetic field is determining the distribution of the initial magnetizations. A previous study showed that, in the case that data are available regarding the magnetic total force anomaly and that the directions of the initial magnetizations are assumed to be uniform, the piezomagnetic field arising from the regional stress is uniquely determined, although the intensities of the initial magnetizations are not uniquely determined. The present study considers a more general situation in which the directions of the initial magnetizations are unknown. The problem addressed here is whether the piezomagnetic field is determined uniquely. To this end, a set of expressions is derived to describe the relation among the initial magnetizations, the magnetic anomalies corresponding to the initial magnetization, and the resultant piezomagnetic field. The expressions show that the correspondence between magnetic anomalies and the piezomagnetic field is not one-to-one. This result suggests that observations of the piezomagnetic field provide quantitative estimations of changes in stress only if we can assume that the directions of the initial magnetizations are uniform.
\end{abstract}

Key words: Regional stress, piezomagnetic effect, direction of magnetization, double Fourier transform, one-toone correspondence.

\section{Introduction}

In the Earth's crust, changes in stress result in changes in magnetization via the piezomagnetic effect (Nagata, 1970). By observing changes in the magnetic field arising from the piezomagnetic effect, which are referred to as piezomagnetic fields, it is possible to monitor the stress accumulation caused by various processes such as slab subduction.

However, studies of the piezomagnetic field encounter difficulties relating to the treatment of the magnetization structures in the Earth's crust. Changes in magnetization are approximately proportional to the product of applied stress and the initial magnetization with no stress (e.g., Stacey, 1964; Nagata, 1970; Stacey and Johnston, 1972). In the absence of constraints on the spatial distribution of the initial magnetization, it is not possible to perform accurate calculations of the piezomagnetic field, even in forward problems. Indeed, numerical examples have demonstrated that significant variations in the magnetic field are observed near areas having strong contrasts in magnetization (e.g., Oshiman, 1990; Nishida et al., 2007) and that, by ignoring the precise structure of the initial magnetization, we obtain an incorrect estimation of the piezomagnetic field (e.g., Currenti et al., 2009).

A steady procedure that can be used to calculate the

Copyright (C) The Society of Geomagnetism and Earth, Planetary and Space Sciences (SGEPSS); The Seismological Society of Japan; The Volcanological Society of Japan; The Geodetic Society of Japan; The Japanese Society for Planetary Sciences; TERRAPUB.

doi: $10.5047 /$ eps.2011.08.007 piezomagnetic field is as follows: first, the spatial distribution of the initial magnetization is assumed by using magnetic survey data; next, the change in magnetization, due to the piezomagnetic effect at each point, is calculated; finally, changes in the magnetic field generated by different parts of the crust are integrated to obtain the magnitude of the entire piezomagnetic field (e.g., Oshiman et al., 1997; Nishida et al., 2004). A drawback of this procedure is the non-uniqueness of solutions in the potential theory. Even in the case that the magnetic field generated by the initial magnetization is completely known, the explicit distribution of the initial magnetizations cannot be determined uniquely. Consequently, the resultant piezomagnetic field is not determined uniquely. This result means that the observed changes in the magnetic field are not directly converted to changes in stress.

Yamazaki (2009) proposed a method of calculating the piezomagnetic field in cases for which (i) the stress field is uniform in the region of interest, and (ii) the directions of initial magnetizations are parallel to the ambient geomagnetic field. Taking into account the similarity between this problem and the reduction to the pole calculation (cf. Baranov, 1957), Yamazaki successfully derived a formula to determine the resultant piezomagnetic field based on magnetic anomaly data. The existence of such a formula implies that the resultant piezomagnetic field is determined uniquely, regardless of the non-uniqueness of the explicit distribution of the initial magnetization intensities. Therefore, the difficulty encountered regarding the 
non-uniqueness of the initial magnetization is avoided for situations in which conditions (i) and (ii) are met. Condition (i) is valid across a reasonably wide range of situations, including stress accumulation related to plate subduction. However, condition (ii) is somewhat problematic. Although this condition is usually assumed in interpretations of aeromagnetic survey results, the directions of the initial magnetizations are not assured to be uniform, particularly in cases of remanent magnetization.

If the uniqueness of the resultant piezomagnetic field is still assured without the need to make assumptions regarding the directions of initial magnetization, the efficiency of studies on the piezomagnetic field would be enhanced. In contrast, if the uniqueness of the resultant piezomagnetic field cannot be assured, we should be cautious in calculating the piezomagnetic field. Therefore, it is important to consider situations in which the directions of the initial magnetizations are unknown. The aim of the present study is to clarify whether the piezomagnetic field is uniquely determined in the case that only data of magnetic anomalies arising from initial magnetizations are available and that no constraints are available regarding the directions of the initial magnetizations.

\section{Definition of the Problem}

It is assumed that changes in magnetization due to the piezomagnetic effect $\left(\Delta J_{i}\right)$ are proportional to the applied stress $\left(\sigma_{i j}\right)$ and the initial magnetization $\left(J_{i}\right)$. The constitutive law is then summarized as follows (Sasai, 1991):

$$
\Delta J_{i}=\frac{3}{2} \beta T_{i j} J_{j}
$$

where $T_{i j}$ is the deviatoric stress tensor given by

$$
T_{i j}=\sigma_{i j}-\frac{1}{3}\left(\sigma_{11}+\sigma_{22}+\sigma_{33}\right),
$$

and $\beta$ is the stress sensitivity. Although the stress sensitivity largely depends on the mechanical strength of the rocks of the crust (e.g. Hamano, 1983), only the situation in which $\beta$ is constant in the region of interest is considered in the present study. In Eq. (1) and hereafter, summation rules for subscripts are used for simplicity of notation. Note that summation rules are not used in the previous paper (i.e. Yamazaki, 2009). Therefore, attention is required when the equations in this paper are compared with those in the previous paper.

A situation is considered in which anomalies in the geomagnetic total force values are known at a certain height (i.e., $\left.x_{3}\right)$. Anomalies in total force values of the geomagnetic field $\left(F_{\mathrm{a}}\right)$ corresponding to crustal magnetizations $\left(J_{i}\right)$ are approximately expressed by the following formula, which is equivalent to equation (5) in Yamazaki (2009):

$$
\begin{aligned}
F_{\mathrm{a}}(\mathbf{x})= & \frac{\mu_{0}}{4 \pi} l_{i} \frac{\partial}{\partial x_{i}} \int_{-\infty}^{\infty} d x_{1}^{\prime} \int_{-\infty}^{\infty} d x_{2}^{\prime} \int_{0}^{H} d x_{3}^{\prime} \\
& \cdot \frac{\partial}{\partial x_{j}} \frac{1}{\left|\mathbf{x}-\mathbf{x}^{\prime}\right|} J_{j}\left(\mathbf{x}^{\prime}\right),
\end{aligned}
$$

where $l_{i}$ denotes the direction cosines of the ambient geomagnetic field and $H$ represents the Curie point depth. A similar expression is obtained for the piezomagnetic field. Using the constitutive law (Eq. (1)), changes in the total forces of the geomagnetic field due to the piezomagnetic effect $\left(F_{\mathrm{p}}\right)$ are expressed by

$$
\begin{aligned}
F_{\mathrm{p}}(\mathbf{x})= & \frac{\mu_{0}}{4 \pi} \frac{3}{2} \beta l_{i} \frac{\partial}{\partial x_{i}} \int_{-\infty}^{\infty} d x_{1}^{\prime} \int_{-\infty}^{\infty} d x_{2}^{\prime} \int_{0}^{H} d x_{3}^{\prime} \\
& \cdot \frac{\partial}{\partial x_{j}} \frac{1}{\left|\mathbf{x}-\mathbf{x}^{\prime}\right|} T_{j k} J_{k}\left(\mathbf{x}^{\prime}\right) .
\end{aligned}
$$

The relations among $J_{i}, F_{\mathrm{a}}$, and $F_{\mathrm{p}}$ are more clearly expressed when considered in wavenumber space. To obtain the double Fourier transforms of Eqs. (3) and (4), the following formula is referred to (e.g., Sasai, 1991):

$$
\begin{aligned}
& \frac{1}{2 \pi} \int_{-\infty}^{\infty} d r_{1} \int_{-\infty}^{\infty} d r_{2} \cdot \frac{\exp \left[-i\left(k_{1} r_{1}+k_{2} r_{2}\right)\right]}{\sqrt{r_{1}^{2}+r_{2}^{2}+r_{3}^{2}}} \\
& =\frac{1}{k_{H}} \exp \left(-k_{H}\left|r_{3}\right|\right),
\end{aligned}
$$

where $k_{H}=\left(k_{1}^{2}+k_{2}^{2}\right)^{1 / 2}$. Using this formula, the double Fourier transforms of $F_{\mathrm{a}}$ and $F_{\mathrm{p}}$ are expressed in the following forms:

$$
F_{\mathrm{a}}^{* *}\left(k_{1}, k_{2}\right)=\frac{\mu_{0}}{4 \pi}\left(l_{i} K_{i}\right)\left(K_{j} J_{j}^{\#}\left(k_{1}, k_{2}\right)\right),
$$

and

$$
F_{\mathrm{p}}^{* *}\left(k_{1}, k_{2}\right)=\frac{\mu_{0}}{4 \pi} \frac{3}{2} \beta\left(l_{i} K_{i}\right)\left(K_{j} T_{j k} J_{k}^{\#}\left(k_{1}, k_{2}\right)\right),
$$

where the superscript ${ }^{* *}$ denotes the double Fourier transform of a function, $K_{i}$ is a scalar multiplier defined by $\left(K_{1}, K_{2}, K_{3}\right)=\left(i k_{1}, i k_{2},-k_{H}\right)$, and the superscript ${ }^{\#}$ denotes the integral defined by

$$
f^{\#}\left(k_{1}, k_{2}\right)=\int_{0}^{H} d x_{3}^{\prime} \cdot f^{* *}\left(k_{1}, k_{2}, x_{3}^{\prime}\right) \exp \left(-k_{H} x_{3}^{\prime}\right) .
$$

Based on Eqs. (6) and (7), it is possible to discuss the relation between $F_{\mathrm{a}}$ and $F_{\mathrm{p}}$. The problem to be solved is whether a given value of $F_{\mathrm{a}}$ uniquely determines the corresponding $F_{\mathrm{p}}$ in the case that the explicit distribution of $J_{i}$ is unknown.

\section{Non-uniqueness of Estimations of the Piezo- magnetic Field}

Before considering general cases, a special case is considered in which the directions of the initial magnetizations are uniform so that the magnetization vector is represented as $J_{i}\left(\mathbf{x}^{\prime}\right)=m\left(\mathbf{x}^{\prime}\right) l_{i}$, where $m$ is a scalar function. In this case, $J_{i}^{\#}$ is expressed as

$$
J_{i}^{\#}\left(k_{1}, k_{2}\right)=m^{\#}\left(k_{1}, k_{2}\right) l_{i} .
$$

By taking the ratio of $F_{\mathrm{p}}^{* *}$ to $F_{\mathrm{a}}^{* *}$, the factor $m^{\#}$ is cancelled. Consequently, $F_{\mathrm{p}}^{* *}$ is uniquely determined by $F_{\mathrm{a}}^{* *}$. The explicit relation is expressed as

$$
F_{\mathrm{p}}^{* *}\left(k_{1}, k_{2}\right)=\frac{3}{2} \beta \frac{l_{j} T_{j k} K_{k}}{l_{i} K_{i}} F_{\mathrm{a}}^{* *}\left(k_{1}, k_{2}\right) .
$$


This formula is equivalent to that derived by Yamazaki (2009), which implies the piezomagnetic field is uniquely determined for a given magnetic anomaly.

In the general situation, the initial magnetizations are not fixed to a single direction. Note that the general situation includes the case of Eq. (9) as a special case. Therefore, if $F_{\mathrm{p}}^{* *}$ is uniquely determined for a given $F_{\mathrm{a}}^{* *}$, the formula of the general situation should also be given by Eq. (10). In particular, $F_{\mathrm{a}}^{* *}=0$ should yield $F_{\mathrm{p}}^{* *}=0$ if Eq. (10) is satisfied.

Unfortunately, a brief inspection of Eqs. (6) and (7) reveals that this expectation is incorrect. For example, consider the following distributions:

$$
\begin{aligned}
\mathbf{J}^{\#}\left(k_{1}, k_{2}\right)= & A \delta\left(k_{1}-k\right) \delta\left(k_{2}\right)\left(\begin{array}{c}
1 \\
0 \\
+i
\end{array}\right) \\
& +A \delta\left(k_{1}+k\right) \delta\left(k_{2}\right)\left(\begin{array}{c}
1 \\
0 \\
-i
\end{array}\right)
\end{aligned}
$$

where $k(>0)$ and $A$ are constants, and $\delta$ is the Dirac Delta function. One of the explicit forms of $\mathbf{J}$ corresponding to Eq. (11) is given by

$$
\mathbf{J}\left(x_{1}^{\prime}, x_{2}^{\prime}, x_{3}^{\prime}\right)=A^{\prime}\left(\begin{array}{c}
\cos k x_{1}^{\prime} \\
0 \\
-\sin k x_{1}^{\prime}
\end{array}\right),
$$

where $A^{\prime}$ is a constant. With reference to Eqs. (6) and $(8), F_{\mathrm{a}}^{* *}$ becomes zero for the above distribution of magnetizations. However, Eq. (7) yields non-zero values of the piezomagnetic field corresponding to this distribution of magnetizations. The initial magnetizations and the changes in magnetizations are illustrated in Fig. 1. This example shows that there are no exact one-to-one correspondences between the magnetic anomalies and the resultant piezomagnetic fields.

Nevertheless, the possibility is that $F_{\mathrm{p}}$ corresponding to a given $F_{\mathrm{a}}$ can be determined in a statistical sense, and that the counterexample given by Eq. (12) is merely an extreme case. This is an analogy to statistical mechanics (e.g. Landau and Lifshitz, 1980). For a given $F_{\mathrm{a}}$, we can consider a set of $J_{i}$, all members of which generate the given $F_{\mathrm{a}}$. If $F_{\mathrm{p}}$ corresponding to major members of the set are approximately the same as those corresponding to the initial magnetizations with a uniform direction (i.e. $F_{\mathrm{p}}$ given by Eq. (10)), then the $F_{\mathrm{p}}$ is regarded as a likely estimation corresponding to the given $F_{\mathrm{a}}$. To confirm whether this expectation is valid or not, it is useful to estimate $F_{\mathrm{p}}$ based on acceptable assumptions regarding $J_{i}$, other than the assumption of a uniform direction of $J_{i}$ (Eq. (9)).

One plausible assumption regarding the initial magnetizations is the minimum length requirement, which is given by

$$
\left|J_{1}^{\#}\left(k_{1}, k_{2}\right)\right|^{2}+\left|J_{2}^{\#}\left(k_{1}, k_{2}\right)\right|^{2}+\left|J_{3}^{\#}\left(k_{1}, k_{2}\right)\right|^{2}=\min .
$$

For a given magnetic anomaly $F_{\mathrm{a}}$, the solution of Eq. (6)

\section{(a) Initial magnetization}

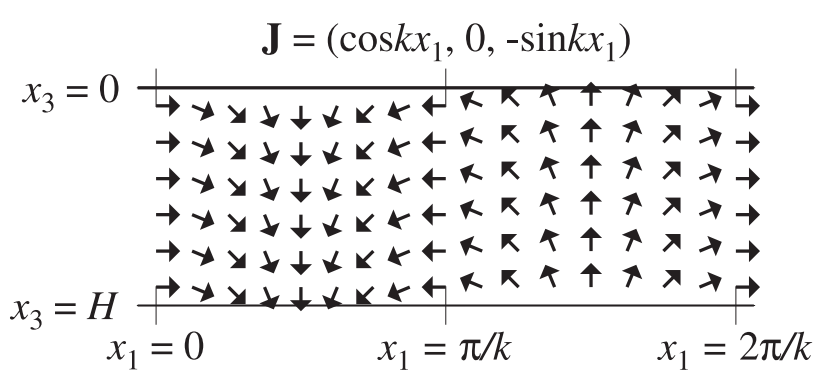

\section{(b) Changes in magnetization}

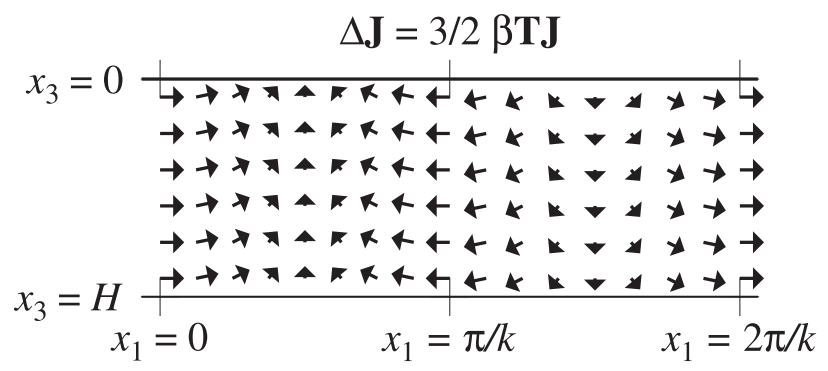

Fig. 1. Example illustrating the non-uniqueness of the piezomagnetic field. (a) Spatial distribution of initial magnetizations ( $\mathbf{J})$ in the crust. $x_{1}$ and $x_{3}$ are the location coordinates, $H$ represents the Curie point depth, and $k$ represents a spatial wavenumber. (b) Changes in the magnetization due to the piezomagnetic effect $(\Delta \mathbf{J})$ corresponding to J. $\beta$ and $\mathbf{T}$ represent the stress sensitivity and a deviatoric stress tensor (i.e., Eq. (2)), respectively. In each panel, arrow length is proportional to the intensity of magnetization. The regional stress field is assumed to be a uniaxial compression with intensity $\sigma$ in the $x_{1}$ direction. The distribution $\Delta \mathbf{J}$ generates a non-zero magnetic field above the ground, whereas $\mathbf{J}$ does not.

that satisfies the requirement of Eq. (13) is given by

$$
J_{i}^{\#}\left(k_{1}, k_{2}\right)=\frac{1}{2 k_{H}^{2}} \frac{4 \pi}{\mu_{0} l_{j} K_{j}} K_{i}^{\prime} F_{\mathrm{a}}^{* *}\left(k_{1}, k_{2}\right),
$$

where $\left(K_{1}^{\prime}, K_{2}^{\prime}, K_{3}^{\prime}\right)=\left(-i k_{1},-i k_{2},-k_{H}\right)$. The corresponding piezomagnetic field is given by

$$
F_{\mathrm{p}}^{* *}\left(k_{1}, k_{2}\right)=\frac{3 \beta}{4 k_{H}^{2}} K_{i} T_{i j} K_{j}^{\prime} F_{\mathrm{a}}^{* *}\left(k_{1}, k_{2}\right) .
$$

The estimations of piezomagnetic fields given by Eqs. (10) and (15) are different, meaning that estimations of the piezomagnetic field differ according to the assumed distributions of the initial magnetizations: an assumption of uniform direction yields Eq. (10), while an assumption of minimum magnetizations yields Eq. (15). Therefore, it is not possible to obtain any estimation of piezomagnetic fields unless the assumption regarding the distribution of the initial magnetization can be demonstrated to be valid.

\section{Conclusions}

In the case of a uniform regional stress, a series of formulae that express the relation between the initial magnetizations, the magnetic anomalies, and the piezomagnetic field, is derived in wavenumber space. Based on the formulae, it is possible to discuss whether there exists a one-to-one correspondence between the magnetic anomalies and the 
piezomagnetic field. In the case that the direction of the initial magnetizations is assumed to be uniform, the piezomagnetic field is uniquely determined from the initial magnetic anomalies, regardless of the non-uniqueness of the explicit distributions of the initial magnetizations. In contrast, in cases for which the directions of the initial magnetization are not uniform, the piezomagnetic field cannot be determined in any sense. This result indicates that the efficiency of piezomagnetic studies is diminished in the case that we cannot assure a uniform direction of initial magnetizations within the region of interest.

The above conclusion is somewhat disappointing because it implies that piezomagnetic fields cannot be correctly determined unless the directions of the initial magnetizations are fixed. The present analysis was limited to the case in which the regional stress is uniform within the region of interest. However, it is natural to expect that the same conclusion would be obtained for a non-uniform regional stress. Of course, this result does not mean that all efforts to observe piezomagnetic fields are meaningless. Observations of the magnetic field will continue to be used for the qualitative detection of events, including changes in the rate of stress accumulation. The present results merely highlight the difficulty encountered in quantitative interpretations of the observed magnetic field. In addition, the directions of the initial magnetizations can be assumed to be uniform in many situations. Indeed, this assumption is adopted in many studies that attempt to interpret the results of magnetic surveys. Nevertheless, we must bear in mind that forward calculations of piezomagnetic fields are accurate only if the direction of magnetization is known.

Acknowledgments. The author is grateful to Dr. C. Del Negro and Dr. Y. Sasai for reviewing the manuscript, and Dr. M. Uyeshima for managing the editorial process.

\section{References}

Baranov, V., A new method for interpretation of aeromagnetic maps: Pseudo-gravimetric anomalies, Geophysics, 22, 359-383, 1957.

Currenti, G., C. Del Negro, A. Di Stefano, and R. Napoli, Numerical simulation of stress induced piezomagnetic fields at Etna volcano, Geophys. J. Int., 179, 1469-1476, 2009.

Hamano, Y., Experiments on the stress sensitivity of natural remanent magnetization, J. Geomag. Geoelectr., 35, 155-172, 1983.

Landau, L. D. and E. M. Lifshitz, Statistical Physics, 3rd Edition, Part 1, 544 pp, Butterworth-Heinemann, Oxford, 1980.

Nagata, T., Basic magnetic properties of rocks under mechanical stresses, Tectonophysics, 9, 167-195, 1970.

Nishida, Y., Y. Sugisaki, K. Takahashi, M. Utsugi, and H. Oshima, Tectonomagnetic study in the eastern part of Hokkaido, NE Japan: Discrepancy between observed and calculated results, Earth Planets Space, 56, 1049-1058, 2004.

Nishida, Y., M. Utsugi, and T. Mogi, Tectonomagnetic study in the eastern part of Hokkaido, NE Japan (II): Magnetic fields related with the 2003 Tokachi-oki earthquake and the 2004 Kushiro-oki earthquake, Earth Planets Space, 59, 1181-1186, 2007.

Oshiman, N., Enhancement of tectonomagnetic change due to non-uniform magnetization in the Earth's crust-Two-dimensional case studies, $J$. Geomag. Geoelectr., 42, 607-619, 1990.

Oshiman, N., Y. Sasai, Y. Honkura, Y. Ishikawa, and Y. Tanaka, Geomagnetic changes correlated with crustal movement in the north-eastern part of the Izu Peninsula, Japan, Annali di Geofisica, 60, 479-494, 1997.

Sasai, Y., Tectonomagnetic modeling on the basis of the linear piezomagnetic effect, Bull. Earthq. Res. Inst., Univ. Tokyo, 66, 587-722, 1991.

Stacey, F. D., The seismomagnetic effect, Pure Appl. Geophys., 58, 5-22, 1964.

Stacey, F. D. and M. J. S. Johnston, Theory of the piezomagnetic effect in titanomagnetite-bearing rocks, Pure, Appl. Geophys., 97, 146-155, 1972.

Yamazaki, K., Calculation of the piezomagnetic field arising from uniform regional stress in inhomogeneously magnetized crust, Earth Planets Space, 61, 1163-1168, 2009.

K. Yamazaki (e-mail: kenichi@rcep.dpri.kyoto-u.ac.jp) 